\title{
CDISC Chronic Hepatitis C Therapeutic Area User Guide Version 1.0
}

National Cancer Institute

\section{Source}

National Cancer Institute. CDISC Chronic Hepatitis C Therapeutic Area User Guide Version 1.0. NCI Thesaurus. Code C161438.

The 1.0 version of the CDISC Chronic Hepatitis C therapeutic area user guide. 